\title{
Análise Sequencial dos comportamentos do terapeuta em psicoterapia com universitários com transtorno de ansiedade social
}

\section{Sequential analysis of the therapist's behavior in psychotherapy with college students with social anxiety disorder \\ El análisis secuencial de la conducta del terapeuta en la psicoterapia con los
estudiantes universitarios con trastorno de ansiedad socials}

Glaucia Fernanda Galeazzi Nobile', Vagner Angelo Garcia², Alessandra Turini Bolsoni-Silva ${ }^{3}$.

[1] [2] [3] Universidade Estadual Paulista (UNESP) I Título abreviado: Análise Sequencial da Interação Terapêutica para o Transtorno de Ansiedade Social I
Endereço para correspondência: Seção Técnica de Pós-Graduação - Faculdade de Ciências. Av. Eng. Luiz Edmundo Carrijo Coube, 14-01. Bairro Vargem
Limpa. Bauru, São Paulo. CEP: $17033-360$ I Email: glau_fgn@hotmail.com I DOI: 10.18761/pac.2016.030

Resumo: O estudo da interação terapêutica possibilita conhecer variáveis interpessoais responsáveis pelas mudanças ocorridas nos comportamentos do cliente e poderá auxiliar na prática de forma a identificar comportamentos do terapeuta que tornam a intervenção mais eficaz. A presente pesquisa teve por objetivo descrever os comportamentos da interação terapêutica, a partir de análises sequenciais, tendo o comportamento do terapeuta como categoria critério. Participaram dois universitários com transtorno de ansiedade social e uma terapeuta. A terapia realizada foi individual, analítico comportamental, incluindo o treino de habilidades sociais. Onze sessões de cada cliente foram categorizadas, utilizando o protocolo de categorias do Sistema Multidimensional de Categorização de Comportamentos na Interação Terapêutica (SiMCCIT) e o software The Observer. Foi realizada análise sequencial tendo o comportamento do terapeuta como critério. O resultado da análise sequencial destaca que os comportamentos do terapeuta de Solicitação de Relato e de Facilitação favoreceram o Relato do cliente, as categorias Informação, Interpretação e Recomendação favoreceram a ocorrência de Concordância do cliente. Com a análise sequencial foi possível encontrar alguns padrões de comportamento do terapeuta e do cliente, sendo um deles, o manejo do terapeuta que favoreceu um ambiente acolhedor e facilitou tanto o relato do cliente como o estabelecimento de relações.

Palavras-chave: interação terapêutica, análise sequencial, transtorno de ansiedade social. 
Abstract: The study of therapeutic interaction enables know interpersonal variables responsible for changes in customer behavior and may help in practice to identify therapist behaviors that make the most effective intervention. This study aimed to describe the behaviors of therapeutic interaction, from sequential analysis, and the therapist's behavior as category criteria. They attended two college with social anxiety disorder and a therapist. The therapy was performed individual, behavioral analytic, including social skills training. Eleven sessions each client were categorized using the Multidimensional System categories protocol behaviors Categorization in Interaction Therapy (SiMCCIT) and The Observer software. Sequence analysis was performed with the therapist's behavior as a criterion. The results of sequential analysis points out that the behavior of the Report Request therapist and Facilitation favored customer report, the categories Information, Interpretation and Recommendation favored the occurrence of Customer Agreement. With the sequential analysis was possible to find some therapist's behavior patterns and customer, one of the therapist's management that favored a warm atmosphere and facilitated both the client's account as the establishment of relations.

Keywords: therapeutic interaction, sequential analysis, social anxiety disorder.

Resumen: El estudio de la interacción terapéutica permite saber las variables interpersonales responsable de los cambios en el comportamiento del cliente y puede ayudar en la práctica para identificar los comportamientos de terapeutas que hacen que la intervención más eficaz. Este estudio tuvo como objetivo describir los comportamientos de interacción terapéutica, a partir del análisis secuencial, y el comportamiento del terapeuta como criterios categoría. Ellos asistieron a la universidad con dos trastorno de ansiedad social y un terapeuta. La terapia se realizó analítica individual, de comportamiento, incluyendo entrenamiento en habilidades sociales. Once sesiones de cada cliente se clasificaron utilizando el Sistema Multidimensional comportamientos de protocolo categorías Categorización de Terapia de Interacción (SiMCCIT) y el software de observador. El análisis de secuencia se realizó con el comportamiento del terapeuta como criterio. Los resultados del análisis secuencial señala que el comportamiento de la Solicitud de informe terapeuta y Facilitación favoreció informe al cliente, las categorías de la Información, Interpretación y Recomendación favorecieron la ocurrencia de acuerdo con el cliente. Con el análisis secuencial fue posible encontrar patrones de comportamiento de algún terapeuta y cliente, uno de gestión del terapeuta que favoreció un ambiente cálido y facilitó tanto la cuenta del cliente como el establecimiento de las relaciones.

Palabras-clave: interacción terapéutica, análisis secuencial, trastorno de ansiedad social. 


\section{Introdução}

Os transtornos de ansiedade têm como característica comum o medo e a ansiedade excessivos. O medo se diferencia da ansiedade, pois o medo é uma resposta a uma ameaça real ou percebida e a ansiedade é a antecipação de uma ameaça futura (APA, 2013). O que diferencia os transtornos de ansiedade é o tipo de objeto ou situação que provoca o medo, ansiedade ou comportamento de esquiva (APA, 2013).

O Transtorno de Ansiedade Social (TAS) de acordo com o Manual Diagnóstico e Estatístico de Transtornos Mentais DSM-V (APA, 2013) é caracterizado quando o indivíduo apresenta medo ou ansiedade frente a situações de interação social. Por exemplo, diante de situações de avaliação por outras pessoas, quando são observadas e quando tem que se apresentar, o indivíduo sente medo em demonstrar os sintomas e ser avaliado negativamente por outros, ou seja, medo da rejeição. Assim as situações sociais são evitadas ou suportadas com sofrimento intenso ocasionando prejuízo nos contextos sociais, ocupacionais e outros importantes da vida da pessoa.

No DSM-V (APA, 2013) ocorreram algumas mudanças em relação ao diagnóstico do Transtorno de Ansiedade Social comparada a quarta edição, DSM - IV TR (APA, 2002). A primeira mudança refere-se à nomenclatura, onde a Fobia Social passou a ser nomeada de Transtorno de Ansiedade Social (TAS). Outra mudança ocorreu em relação ao critério de duração, que passou a ter duração mínima de seis meses para todas as idades, sendo que na versão anterior, tal critério era exigido apenas para crianças. Também não é mais exigido para o diagnóstico que o indivíduo avalie seu medo como excessivo ou irracional, já que existe a possibilidade de superestimar o perigo do evento fóbico (Araújo $\&$ Neto, 2014).

Batista et al. (2012) avaliaram a prevalência do Transtorno de Ansiedade Social em uma amostra de 2319 estudantes brasileiros universitários com o objetivo de determinar o impacto acadêmico do transtorno. Foram aplicados na fase de triagem o Inventário de Fobia Social (SPIN) e a MINI-SPIN e na fase de confirmação diagnóstica foi aplicado o SCID-IV. O estudo mostrou a prevalência do trans- torno em estudantes universitários de 11,6\%, com início precoce, aproximadamente aos 11 anos de idade. Sendo no sexo feminino a maior incidência, e o medo de falar em público, o mais comum.

Garcia-Lopez, Salvador e Reyes (2015) discorrem sobre a importância da avaliação do Transtorno de Ansiedade Social em adolescentes para um diagnóstico com maior confiabilidade. Os autores ressaltaram a importância da avaliação clínica com entrevista para avaliar a vida social do adolescente, sendo importante também a participação dos pais nessa avaliação, na medida em que o adolescente pode desvalorizar os sintomas. Outro fator importante para a avaliação nos casos de TAS, apresentada pelos autores é a interação terapêutica.

O termo interação terapêutica, na perspectiva da análise do comportamento pode ser descrito enquanto análise do comportamento verbal do terapeuta e do cliente. Segundo Skinner (1957), o comportamento verbal é um comportamento operante, cuja consequência para o comportamento de quem fala é produzida pelo comportamento de quem ouve. Assim, a classificação dos comportamentos da interação terapêutica levará em conta a função do comportamento do terapeuta e do cliente. Tal análise possibilita estudar importantes processos, como a tomada de decisão do terapeuta, consequências disponibilizadas pelo terapeuta às ações do cliente, manejo de situações e orientação (Zamignani, 2007). No tratamento do TAS, assim como em qualquer outra psicoterapia, é importante esse conhecimento para saber o que possibilita que a terapia alcance ou não seu êxito.

Nesse sentido, para avaliar a eficácia e efetividade de intervenções, de acordo com a Psicologia Baseada em Evidência (PBE), é importante avaliar tanto medidas de resultado quanto de processo, sendo a interação terapêutica uma forma de medida de processo (APA, 2006). A PBE é definida pela integração das melhores evidências em pesquisas disponíveis com a experiência clínica, considerando a cultura, as características e preferências do paciente (APA, 2006).

Nesta perspectiva, o estudo da interação terapêutica na intervenção para o TAS, com a investigação dos processos de mudança no tratamento contribui com a $\mathrm{PBE}$, pois produz conhecimento que pode aperfeiçoar a prática do terapeuta e forne- 
cer ao cliente um tratamento eficaz. Sendo intuito deste trabalho, contribuir para a construção desse conhecimento, a seguir são apresentados estudos de intervenção com fóbicos sociais.

\section{Intervenções para o TAS}

O estudo apresentado por Rocha, Bolsoni-Silva e Verdu (2012) teve como objetivo avaliar os efeitos de um procedimento de intervenção comportamental associado a treinamento de habilidades sociais em quatro universitários com o diagnóstico de TAS sem comorbidades. Ocorreram 12 encontros semanais em atendimento individual com duração de duas horas. A intervenção adotada foi desenvolvida por Bolsoni-Silva (2009) e aplicada em contexto de grupo. Foi utilizado delineamento de linha de base múltipla e os instrumentos foram aplicados antes, após a intervenção e em seguimento de quatro meses. Os resultados do estudo mostraram que ao final da intervenção os participantes deixaram de apresentar o diagnóstico de fobia social e mostraram aumento no repertório de habilidades sociais e redução de dificuldades interpessoais. As autoras concluíram que a Terapia Comportamental combinada com o Treinamento de Habilidades Sociais permitiu que os participantes identificassem as variáveis de controle dos seus comportamentos e que desenvolvessem estratégias de melhoria dos relacionamentos.

Beidel et al. (2014) investigaram o impacto de uma intervenção com treinamento de habilidades sociais para o tratamento do Transtorno de Ansiedade Social (TAS). A amostra contou com 106 participantes adultos diagnosticados com TAS, que foram divididos em três grupos: I - apenas de terapia de exposição; II - com combinação de treinamento de habilidades sociais e III - lista de espera (grupo controle). Os dois grupos de intervenção apresentaram melhora significativa em relação ao grupo controle, no entanto, a intervenção com combinação de habilidades sociais (II) produziu resultados superiores, tendo maior redução no estado clínico em medidas de avaliação da ansiedade social e em medidas de comportamento social, sendo essa diferença mantida em seis meses de seguimento. Os autores concluíram que o Treinamento de Habilidades Sociais mostrou-se importante na otimização dos resultados no tratamento, pois promoveu mudanças de comportamentos consistentes nos clientes com TAS.

Barkowski et al. (2016) em um estudo de revisão de literatura investigou a eficácia da psicoterapia em grupo para pessoas adultas com TAS e comparou com outros tipos de tratamento, como a terapia individual e a medicamentosa. Um dos critérios de revisão foram pesquisas publicadas a partir de 1990 até 2015, sendo identificados 36 estudos, com a maioria (34 estudos) utilizando a Terapia Cognitiva Comportamental em Grupo (TCCG). A terapia em grupo se apresentou superior ao grupo controle e melhorou os sintomas do TAS. Contudo, não foram encontradas diferenças entre a terapia individual e de grupo, sendo possível concluir que a terapia em grupo TCCG é eficaz para o tratamento de TAS, assim como o tratamento individual.

Esses estudos de intervenções para o TAS mostraram, através de medidas de resultado, que a Terapia Cognitiva Comportamental, Terapia Analítico Comportamental e o Treino de Habilidades Sociais promovem a diminuição dos sintomas característico do TAS. De acordo com a $\mathrm{PBE}$, as pesquisas preocupadas em avaliações processuais e em resultados de processo também são importantes para a avaliação da efetividade e eficácia, pois permitem identificar elementos da interação terapêutica que garantem a obtenção dos resultados desejados. Assim, o estudo da interação terapêutica possibilita uma maior compreensão dos processos de mudança, contribuindo para o conhecimento na área clínica, juntamente com pesquisas de medidas de resultado e de avaliação de procedimento.

\section{Estudos da interação terapêutica}

Nas pesquisas recentes sobre a interação terapêutica na perspectiva analítico comportamental, muitas pesquisas vêm se destacando. Uma delas trata-se da tese de livre docência de Meyer (2009), em que foi criado um banco de dados de sessões de terapia comportamental. A autora propõe uma nova organização de categorias comportamentais do terapeuta e analisa as categorias Solicitação de Informação e Recomendação. 
Zamignani (2007) após revisão de literatura sobre a interação terapêutica na abordagem comportamental, elaborou um Sistema Multidimensional de Categorização de Comportamentos da Interação Terapêutica (SiMCCIT), para categorização dos comportamentos do terapeuta e do cliente. O SiMCCIT é composto por três eixos de categorização: Eixo I - comportamento verbal, Eixo II - temas e Eixo III - respostas motoras, sendo que os Eixos I e II possuem também qualificadores, que especificam propriedades do comportamento categorizado. O SiMCCIT oferece um catálogo amplo de fácil utilização, sendo relevante na utilização para o ensino de habilidades básicas do terapeuta (Zamignani \& Meyer, 2011).

Pesquisas de interação terapêutica na Análise do Comportamento (utilizando o SiMCCIT), podem organizar os dados em termos de frequência (ocorrência) e duração (segundo). A partir desse ponto, podem ser elaborados outros tipos de análise, como estudos de correlação e também de análise sequencial (Sadi, 2011; Silveira, Bolsoni-Silva, \& Meyer, 2010; Xavier, Kanter, \& Meyer, 2012).

Silveira et al. (2010) descreveram padrões de interação entre terapeuta e cliente em uma intervenção de grupo com Treinamento de Habilidades Sociais. Para a categorização foi utilizado o eixo I do SiMCCIT (Zamignani, 2007) com o auxílio do software The Observer XT 7.0, sendo analisadas as filmagens de cinco sessões. Foi realizada análise sequencial, e os resultados apresentaram a categoria Recomendação ligada à categoria Aprovação. Esse dado sugere que o terapeuta ao utilizar de Aprovação seguido por Recomendação, o fez de forma a reduzir possíveis efeitos adversos da categoria Recomendação, bem como, incentivar ao cliente seguir a Recomendação. As autoras ainda apontam direcionamentos para aperfeiçoar a metodologia utilizada em seu estudo, salientando que devem ser realizadas mais pesquisas com análises sequenciais, utilizando também outros eixos do SiMCCIT (Zamignani, 2007), além de pesquisas com clientes com outros diagnósticos.

No estudo de Sadi (2011) identificaram-se as variáveis relacionadas ao abandono da terapia em um caso de Transtorno de Personalidade Borderline. Este estudo utilizou o Eixo I e o Eixo II do SiMCCIT (Zamignani, 2007), analisando o áudio de 13 sessões de uma díade terapêutica. Além da descrição em termos de frequência e duração, foi também realizada análise sequencial de atraso. Entre os resultados, verifica-se que o tema com maior frequência e duração foi Queixas psiquiátricas e sintomas médicos, e as sequências de interações de comportamentos do terapeuta/cliente com maiores frequências foram Relato seguido por Facilitação e Facilitação seguida por Relato. Sadi (2011) conclui que o abandono esteve relacionado com diversos fatores entre eles a perda de oportunidade do terapeuta em aprovar, solicitar reflexão e interpretar.

Xavier et al (2012) descreveram o processo de modelagem de repertório de dois casos de Terapia Analítico Comportamental Infantil. Para a categorização dos comportamentos do cliente foi utilizado o Functional Analytic Psychotherapy Rating Scale (FAPRS) e para os comportamentos do terapeuta, o SiMCCIT. A categorização foi realizada através do software The Observer Pro. Foram analisadas 10 sessões de duas terapias com uma criança cada, sendo a queixa relacionada a dificuldades escolares. Uma das terapias foi bem sucedida com duração de 18 sessões, sendo que a outra, ainda estava em curso, e foram utilizadas sessões do primeiro ano de atendimento. Ambas as terapias eram baseadas no referencial FAP (Psicoterapia Analítica Funcional), que inclui em seus conceitos, o CCR (comportamento clinicamente relevante). A análise de probabilidade de transição foi usada para verificar se ocorria comportamento diferencial do terapeuta de acordo com o comportamento clinicamente relevante do cliente. Os resultados da análise de probabilidade de transição apontaram regularidades do comportamento do terapeuta após os comportamentos clinicamente relevantes do cliente, com destaque para a Aprovação após CCR2. Os autores concluíram que a utilização da probabilidade de transição para a análise de comportamentos categorizados mostrou-se relevante para o estudo da interação terapêutica.

Em dois estudos do grupo de pesquisadores Ruiz-Sancho, Frojan-Parga e Calero-Elvira (2013, 2015), foi realizada análise sequencial de $19 \mathrm{ca}-$ sos de intervenção comportamental, que compreendiam 92 sessões de terapia. Ruiz-Sancho et al. (2013) tiveram como objetivo entender o processo 
de aprendizagem que pode ser responsável pelas mudanças de comportamento do cliente, dessa forma, o estudo buscou confirmar a premissa de que o terapeuta responde diferencialmente ao cliente, expressando respostas de aprovação para conteúdos que tinham relação com os objetivos terapêuticos, dado que foi confirmado nos resultados.

Ruiz-Sancho et al (2015), por sua vez, buscou analisar a relação do comportamento do terapeuta com o comportamento do cliente através da análise sequencial. Os resultados mostraram a ocorrência de padrões da interação verbal relacionados com os comportamentos clinicamente relevantes. Por exemplo, quando ocorre uma verbalização longa de explicação, a fala é interrompida pelo próprio terapeuta para verificar o entendimento do cliente. Os autores concluem que a utilização da análise sequencial é adequada para o estudo da mudança clínica, e ainda destaca a importância da análise funcional da interação para explicar o processo terapêutico.

Raustan, Rodríguez \& Argilaga (2013) analisaram a interação terapêutica com o objetivo de identificar padrões sequenciais de comportamentos. A intervenção ocorreu com um grupo de sete mulheres sobreviventes do câncer de mama, e a terapeuta possuía experiência na abordagem analítica de grupo. Nove sessões foram transcritas e realizada analise sequencial, tendo o comportamento do terapeuta como comportamento critério. Os resultados mostraram que o terapeuta mantinha comportamentos empáticos diante de relatos de apoio emocional por parte dos integrantes do grupo; o terapeuta também se mantinha em silêncio diante da fala do grupo, demonstrando atenção e estimulando o grupo a também não interromper a fala de outro integrante. Os autores concluem que o comportamento do terapeuta é o eixo fundamental no qual ocorrem as interações e facilita a mudança terapêutica.

Um estudo recente de interação terapêutica na análise do comportamento com universitários com TAS é o de Garcia (2014). Em sua pesquisa foram realizados três estudos: I - o objetivo foi a descrição, em frequência e duração, das categorias dos comportamentos do terapeuta e cliente; II - a descrição, em frequência e duração, dos temas das sessões e III - realizada a análise de correlação en- tre as categorias dos comportamentos do terapeuta, do cliente e do tema da sessão.Garcia (2014) utilizou 22 sessões de dois clientes que passaram por intervenção comportamental com treino de habilidades sociais, descrito em Rocha (2012). O Estudo III aponta a correlação das categorias do terapeuta Solicitação de Relato com as categorias Facilitação e Gestos de concordância, que sugere que o terapeuta apresentou escuta ativa. A correlação de Empatia com Informações e Recomendações, mostra que o terapeuta foi empático ao informar e recomendar ao cliente. Para as categorias do cliente, a correlação de Relações com Relato, Metas e Concordância, evidencia a ocorrência de análise funcional realizada pelo cliente e ainda a formulação de metas para a solução de seus problemas.

Estudos (Garcia, 2014; Silveira, et al, 2010; Xavier, et al, 2012) ressaltaram que pesquisas futuras realizassem análise sequencial dos comportamentos da díade terapêutica, como forma de buscar a compreensão e descrição de possíveis relações de dependência. Assim, a presente pesquisa tem como objetivo descrever padrões de comportamentos da interação terapêutica em uma terapia analítico comportamental para casos de transtorno de ansiedade social, a partir de análises sequenciais tendo o comportamento do terapeuta como categoria critério.

\section{Método}

\section{Participantes}

Dois estudantes universitários diagnosticados com TAS sem comorbidades e uma terapeuta. Os clientes realizaram terapia individual (analítico comportamental) associado ao Treino de Habilidades Sociais (Rocha, 2012). Cliente 1 - P1: homem com 19 anos, cursando $2^{\circ}$ ano de Bacharelado em Ciência da Computação, solteiro/sem namorada, não trabalhava e residia em república com um amigo. Cliente 2 - P2: mulher com 22 anos, cursando o $4^{\circ}$ ano de Pedagogia, solteira/com namorado, realizava estágio curricular e residia em uma república com quatro amigas.

Os participantes passaram por 12 sessões de terapia, que foram filmadas e tinham aproximadamente duas horas de duração cada. Essa inter- 
venção foi considerada de sucesso, pois os clientes apresentaram aumento do repertório de comportamentos socialmente habilidosos e deixaram de ter o diagnóstico após a intervenção e em seguimento (Rocha, 2012; Rocha et al., 2012). A pesquisa possui a aprovação do Comitê de Ética e os participantes (P1, P2 e a terapeuta) assinaram o Termo de Consentimento Livre e Esclarecido autorizando a utilização das filmagens.

\section{Material}

Foram utilizados os arquivos em vídeos das gravações das sessões para a categorização dos comportamentos. A intervenção foi realizada em 12 sessões, mas foram utilizadas para categorização 11 sessões gravadas em vídeos para cada cliente, 22 sessões no total. Cada sessão contém aproximadamente duas horas de duração, totalizando 38 horas e 15 minutos.

O Eixo I do SiMCCIT foi utilizado como Protocolo de Observação para a realização das categorizações das sessões, referente aos comportamentos verbal vocal e não vocal do terapeuta e do cliente, sendo 15 categorias referentes ao terapeuta e 13 ao cliente, que estão citadas na tabela 1 (Zamignani, 2007).

Tabela 1. Categorias do SiMCCIT (Zamignani, 2007):

\begin{tabular}{ll}
\hline Terapeuta & Cliente \\
\hline Solicita relato (SRE) & Solicitação (SOL) \\
Facilitação (FAC) & Relato (REL) \\
Empatia (EMP) & Melhora (MEL) \\
Informações (INF) & Metas (MET) \\
Solicita reflexão (SRF) & Estabelece relações \\
Recomendação (REC) & (CER) \\
Interpretação (INT) & Concordância (CON) \\
Aprovação (APR) & Oposição (OPO) \\
Reprovação (REP) & Outras verbalizações do \\
Outras verbalizações do & cliente (COU) \\
terapeuta (TOU) & Registro Insuficiente (CIN) \\
Registro insuficiente (TIN) & Gestos de concordância \\
Gestos de concordância & cliente (CCN) \\
terapeuta (GCT) & Gestos de discordância \\
Gestos de discordância & Cliente (DC) \\
terapeuta (GDT) & Respostas não vocais de \\
Respostas não vocais de & pedido/ordem/comando/ \\
pedido/ordem/comando/ & incentivo (COM) \\
incentivo (GMT) & Gestos outros cliente \\
Gestos outros (GOT) & (GCO) \\
&
\end{tabular}

Obtendo índices satisfatórios de concordância ocorreu a quarta fase, em que foram categorizadas
Foi utilizado o software Clic $^{\oplus}$ para a realização do treino sistemático de observadores, desenvolvido por Zamignani (2007), com a finalidade do uso adequado do SiMCCIT e o software The Observer $X T 7.0$ para a categorização das sessões através da utilização de recurso audiovisual (áudio e vídeo).

\section{Procedimento e análise dos dados}

As sessões foram categorizadas por dois observadores, sendo ambos graduados em Psicologia, com um ano de experiência em terapia comportamental por meio de estágio curricular. O tratamento dos dados foi dividido em cinco fases.

$\mathrm{Na}$ primeira fase foram realizados os seguintes procedimentos: (a) o estudo do SiMCCIT de Zamignani (2007); (b) treino sistemático de observadores através do software $\mathrm{Clic}^{\oplus}$; (c) registro das categorias e a introdução dos vídeos das sessões no software The Observer XT 7.0.

$\mathrm{Na}$ segunda fase foi efetuado o consenso entre observadores, com o objetivo de avaliar o nível de concordância entre os observadores. Para tanto foram utilizadas duas medidas, sendo uma delas o percentual de concordância fornecida pelo software The Observer XT 7.0, e a outra o coeficiente Kappa.

$\mathrm{Na}$ terceira fase foi avaliada a concordância entre os observadores sem haver comunicação entre eles. Nessa fase foram categorizadas $20 \%$ do total de sessões para análise por ambos os observadores (4,4 sessões: aproximado para 5 sessões do total de 22 sessões). As sessões foram sorteadas aleatoriaresultados satisfatórios dos índices de concordância:

- P1 sessão 01: Concordância 81\%, Coeficiente Kappa 0,79;

- P1 sessão 10: Concordância 79\%, Coeficiente Kappa 0,78;

- P1 sessão 11: Concordância $81 \%$, Coeficiente Kappa 0,80;

- P2 sessão 03: Concordância 81\%, Coeficiente Kappa 0,80;

- P2 sessão 12: Concordância 85\%, Coeficiente Kappa 0,84. mente. A seguir estão as sessões sorteadas com os 
as 17 sessões faltantes que não entraram na terceira fase de concordância. Essas sessões foram divididas entre observadores, que utilizar do som o software The Observer XT 7.0 para a categorização.

Por fim, a quinta fase abrange a análise sequencial, que foi realizada através de função lag sequential analysis do software The Observer XT 7.0, que consiste em determinar a probabilidade de um evento preceder ou suceder outro evento, dessa forma é possível observar regularidades em padrões de sequências de comportamentos (Bakerman \& Gottman, 1997).

Para a análise sequencial foram sorteadas três sessões, sendo as de número 2,6 e 10, repetidas de cada cliente (total de seis sessões para análise). A partir da categoria critério foi realizada análise em seis níveis: três antecedentes, "Lag -1", "Lag -2", "Lag -3", e subsequentes, "Lag +1", "Lag +2" e "Lag +3 ".

As categorias escolhidas como categoria critério foram selecionadas do estudo de Garcia et al (2015), sendo elas: (a) as que obtiveram maior frequência (Facilitação e Informação); (b) maior dura- ção (Informação, Interpretação e Recomendação); (c) as categorias que diferenciaram os clientes (Facilitação e Interpretação); (d) e as categorias relacionadas à melhora do cliente (Aprovação).

\section{Resultados}

Os resultados da pesquisa foram organizados em forma de tabelas, sendo uma tabela para cada categoria critério. A Tabela 2 descreve as análises sequencias da categoria critério Solicitação de Relato (SRE), a Tabela 3 descreve as análises sequenciais da categoria critério Facilitação (FAC), a Tabela 4 descreve as análises sequenciais da categoria critério Informação (INF), a Tabela 5 descreve as análises sequenciais da categoria critério Interpretação (INT), a Tabela 6 descreve as análises sequenciais da categoria critério Recomendação (REC) e a Tabela 7 descreve as análises sequenciais da categoria critério Aprovação (APR). Nas Tabelas de resultados foram incluídas apenas as categorias que obtiveram maior probabilidade condicional em relação às categorias critério.

Tabela 2. Análises Sequenciais das sessões 2, 6 e 10, para os dois clientes, em seis níveis, Lag -1, Lag - 2, Lag -3, Lag+1, Lag +2 e Lag +3. A categoria de comportamento do terapeuta selecionada como evento critério é Facilitação (FAC). As categorias do terapeuta estão grifadas.

\begin{tabular}{|c|c|c|c|c|c|c|c|c|c|c|c|c|c|c|c|c|c|c|}
\hline \multicolumn{3}{|c|}{ Lag -3} & \multicolumn{3}{|c|}{ Lag -2} & \multicolumn{3}{|c|}{ Lag -1} & \multirow{2}{*}{$\begin{array}{l}\text { Evento } \\
\text { Critério }\end{array}$} & \multicolumn{3}{|c|}{$\operatorname{Lag}+1$} & \multicolumn{3}{|c|}{ Lag +2} & \multicolumn{3}{|c|}{ Lag +3} \\
\hline Cat. & Fre. & $\mathrm{PC}$ & Cat. & Fre. & PC & Cat. & Fre. & $\mathrm{PC}$ & & Cat. & Fre. & $\mathrm{PC}$ & Cat. & Fre. & PC & Cat. & Fre. & PC \\
\hline \multicolumn{19}{|c|}{ SESSÃO 02} \\
\hline$\frac{\text { SRE }}{\mathrm{CON}}$ & 15 & 0,18 & $\underline{\mathrm{SRE}}$ & 24 & 0,29 & $\frac{\text { FAC }}{\text { REL }}$ & 36 & 0,43 & $\underline{F A C}$ P1 & $\underline{F A C}$ & 36 & 0,43 & CON & 15 & 0,18 & $\mathrm{CON}$ & 21 & 0,25 \\
\hline REL & 8 & 0,21 & $\underline{\mathrm{SRE}}$ & 13 & 0,34 & REL & 15 & 0,39 & FAC P2 & $\underline{F A C}$ & 10 & 0,26 & $\begin{array}{l}\text { REL } \\
\text { CER }\end{array}$ & 8 & 0,21 & CER & 7 & 0,18 \\
\hline \multicolumn{19}{|c|}{ SESSÃO 06} \\
\hline FAC & 46 & 0,25 & FAC & 40 & 0,22 & FAC & 83 & 0,45 & $\underline{\text { FAC P1 }}$ & FAC & 83 & 0,45 & $\underline{F A C}$ & 40 & 0,22 & FAC & 46 & 0,25 \\
\hline REL & 9 & 0,25 & SRE & 14 & 0,39 & $\underline{\mathrm{REL}}$ & 19 & 0,53 & FAC P2 & $\underline{\mathrm{SRE}}$ & 14 & 0,39 & REL & 14 & 0,39 & SRE & 10 & 0,28 \\
\hline \multicolumn{19}{|c|}{ SESSÃO 10} \\
\hline FAC & 12 & 0,22 & SRE & 15 & 0,28 & REL & 30 & 0,56 & $\underline{\text { FAC P1 }}$ & $\frac{\text { FAC }}{\text { APR }}$ & 12 & 0,22 & $\mathrm{CON}$ & 16 & 0,30 & $\frac{\mathrm{FAC}}{\mathrm{CON}}$ & 12 & 0,22 \\
\hline REL & 9 & 0,22 & SRF & 13 & 0,32 & CER & 14 & 0,34 & FAC P2 & $\begin{array}{l}\text { SRE } \\
\text { FAC }\end{array}$ & 12 & 0,29 & REL & 13 & 0,32 & $\underline{\text { SRE }}$ & 8 & 0,20 \\
\hline
\end{tabular}

Tabela 1: Categoria (Cat.), Frequência (Fre.), Probabilidade Condicional (PC), Solicita relato (SRE), Facilitação (FAC), Solicita reflexão (SRF), Aprovação (APR), Relato (REL), Estabelece relações (CER), Concordância (CON). 
Conforme a Tabela 2 não ocorre com grande regularidade uma sequência de comportamentos para todas as sessões e para os dois clientes, mas pode-se destacar com alta ocorrência a categoria Facilitação após a categoria critério Facilitação. Outra regularidade é a categoria Solicita Relato que aparece para os dois clientes com destaque na Lag -2.

Ao se comparar os clientes P1 e P2, nota-se pouca semelhança. Destaca-se na sessão 1 a Lag
-2 com a categoria Solicita Relato e Lag +1 com a categoria Facilitação; na sessão 10 a Lag -2 com a categoria Solicita Reflexão. Para o cliente P1 em todas as sessões ocorre a categoria Facilitação em alguma Lag, com destaque para a sessão 6 que na sequencia das seis Lags ocorreu Facilitação. As categorias que mais apareceram nas sequências foram, Solicita Relato, Relato, Facilitação e Concordância.

Tabela 3. Análises Sequenciais das sessões 2, 6 e 10, para os dois clientes, em seis níveis, Lag -1, Lag - 2, Lag -3, Lag+1, Lag +2 e Lag +3. A categoria de comportamento do terapeuta selecionada como evento critério é Informação (INF). As categorias do terapeuta estão grifadas.

\begin{tabular}{|c|c|c|c|c|c|c|c|c|c|c|c|c|c|c|c|c|c|c|}
\hline \multicolumn{3}{|c|}{ Lag -3} & \multicolumn{3}{|c|}{ Lag -2} & \multicolumn{3}{|c|}{ Lag -1 } & \multirow{2}{*}{$\begin{array}{l}\text { Evento } \\
\text { Critério }\end{array}$} & \multicolumn{3}{|c|}{ Lag +1 } & \multicolumn{3}{|c|}{ Lag +2} & \multicolumn{3}{|c|}{ Lag +3} \\
\hline Cat. & Fre. & PC & Cat. & Fre. & $\mathrm{PC}$ & Cat. & Fre. & PC & & Cat. & Fre. & PC & Cat. & Fre. & $\mathrm{PC}$ & Cat. & Fre. & $\mathrm{PC}$ \\
\hline \multicolumn{19}{|c|}{ SESSÃO 02} \\
\hline CON & 12 & 0,21 & CON & 21 & 0,38 & CON & 16 & 0,29 & INF P1 & CON & 29 & 0,52 & CON & 18 & 0,32 & CON & 16 & 0,29 \\
\hline INT & 3 & 0,15 & $\underline{\mathrm{INF}}$ & 6 & 0,30 & $\underline{\underline{\mathrm{INT}}}$ & 4 & 0,20 & INF P2 & $\frac{\frac{\mathrm{SRE}}{\mathrm{SRF}}}{\frac{\mathrm{REC}}{\mathrm{REL}}}$ & 3 & 0,15 & $\underline{\mathrm{INF}}$ & 6 & 0,30 & FAC & 5 & 0,25 \\
\hline \multicolumn{19}{|c|}{ SESSÃO 06} \\
\hline INF & 9 & 0,26 & CON & 9 & 0,26 & CON & 11 & 0,31 & INF P1 & CON & 15 & 0,43 & CON & 12 & 0,34 & $\mathrm{CON}$ & 10 & 0,29 \\
\hline REL & 6 & 0,26 & $\underline{\mathrm{INF}}$ & 5 & 0,22 & APR & 7 & 0,30 & INF P2 & $\underline{R E C}$ & 6 & 0,26 & $\underline{\mathrm{INF}}$ & 5 & 0,22 & $\frac{\frac{\mathrm{FAC}}{\mathrm{INF}}}{\underline{\mathrm{REC}}}$ & 4 & 0,17 \\
\hline \multicolumn{19}{|c|}{ SESSÃO 10} \\
\hline CON & 4 & 0,21 & $\begin{array}{l}\frac{\mathrm{REC}}{\mathrm{REL}} \\
\mathrm{CON}\end{array}$ & 3 & 0,16 & CON & 4 & 0,21 & INF P1 & CON & 10 & 0,53 & CON & 5 & 0,26 & $\begin{array}{l}\frac{\mathrm{SRF}}{\mathrm{REL}} \\
\mathrm{CON}\end{array}$ & 3 & 0,16 \\
\hline REL & 5 & 0,24 & $\underline{\mathrm{INF}}$ & 6 & 0,29 & $\frac{\text { FAC }}{\text { EMP }}$ & 3 & 0,14 & INF P2 & $\frac{\mathrm{APR}}{\mathrm{CON}}$ & 4 & 0,19 & $\underline{\mathrm{INF}}$ & 6 & 0,29 & $\underline{I N F}$ & 4 & 0,19 \\
\hline
\end{tabular}

Tabela 2: Categoria (Cat.), Frequência (Fre.), Probabilidade Condicional (PC), Solicita relato (SRE), Facilitação (FAC), Empatia (EMP), Informações (INF), Solicita reflexão (SRF), Recomendação (REC), Interpretação (INT), Aprovação (APR), Relato (REL), Concordância $(\mathrm{CON})$.

De acordo com a Tabela 3 a categoria critério Informação apresenta como regularidade em $\mathrm{P} 1 \mathrm{a}$ categoria Concordância como subsequente e antecedente, já para o cliente P2 não ocorre uma categoria específica nem como antecedente, nem subsequente, por exemplo, na primeira sessão a categoria Informação é sucedida por várias categorias com a mesma probabilidade condicional com valor baixo. De modo geral para o cliente P2 as categorias possuem uma probabilidade condicional baixa.

Comparando as sessões, aparece uma regularidade nas sessões 2 e 6 para P1, em que aparece em sequencia a categoria Concordância como antecedente e subsequente, já para $\mathrm{P} 2$ as sessões 2, 6 e 10 aparecem na Lag-2 e Lag+2 a categoria Informação.

$\mathrm{Na}$ Tabela 4 que apresenta a categoria critério Interpretação é possível notar regularidade para o cliente P1, mas não para P2. Em P1 a categoria Concordância aparece como subsequente em todas as Lags da categoria critério e em todas as sessões, com exceção da sessão 6 em Lag +3 que ocorre a categoria Facilitação. Em P2 as categorias possuem uma probabilidade condicional baixa, o que não configura dados significativos para a análise, con- 
tudo é possível identificar que em todas as sessões a categoria Facilitação ocorre como antecedente para Interpretação, diferentemente de $\mathrm{P} 1$, para a cliente P2 a categoria Estabelece Relações do cliente ocorre como antecedente em algum nível de Lag tanto para a sessão 2 quanto para a sessão 6, já em níveis consequentes ocorrem muitas categorias com probabilidade condicional baixa, não mostrando um padrão de comportamento entre os comportamentos do terapeuta e cliente.

Conforme a Tabela 5 que apresenta a categoria critério Recomendação, há a presença de várias categorias como antecedentes e como subsequentes. $\mathrm{Na}$ sessão 6 tanto para P1 quanto para P2 a categoria Informação ocorre como antecedente e na sessão 10 a categoria Concordância ocorre como consequente para os dois clientes.

A Tabela 5 evidencia que a categoria Concordância ocorre com maior frequência nas sequências do cliente P1 do que em P2, sendo que em todas as sessões ocorre como subsequente Lag+1, na sessão 2 e 6 como Lag+1 e Lag+2. Para P2 as categorias Recomendação e Informação são antecedentes de Recomendação. Na sessão 10 para P2 apenas ocorre Recomendação como antecedente e subsequente de Recomendação.

De acordo com a Tabela 6 as sessões 2 e 6 mostram-se semelhantes tanto para P1 quanto para P2, sendo que para o cliente P1 a categoria critério Aprovação tem como antecedente as categorias Facilitação e Concordância, e como subsequente a categoria Concordância. Por sua vez, para o cliente P2, as categorias Solicitação de Relato e Relato aparecem como antecedentes e as categorias Informação e Facilitação como subsequentes. Para o cliente $\mathrm{P} 1$ destaca-se que nas três sessões e nos três níveis de Lag, ocorre como subsequente a categoria Concordância. Outro destaque é que na sessão 10 para $\mathrm{P} 2$, aparece como antecedente à Aprovação, as categorias Facilitação e Empatia.

Tabela 4. Análises Sequenciais das sessões 2, 6 e 10, para os dois clientes, em seis níveis, Lag -1, Lag - 2, Lag -3, Lag+1, Lag +2 e Lag +3. A categoria de comportamento do terapeuta selecionada como evento critério é Interpretação (INT). As categorias do terapeuta estão grifadas.

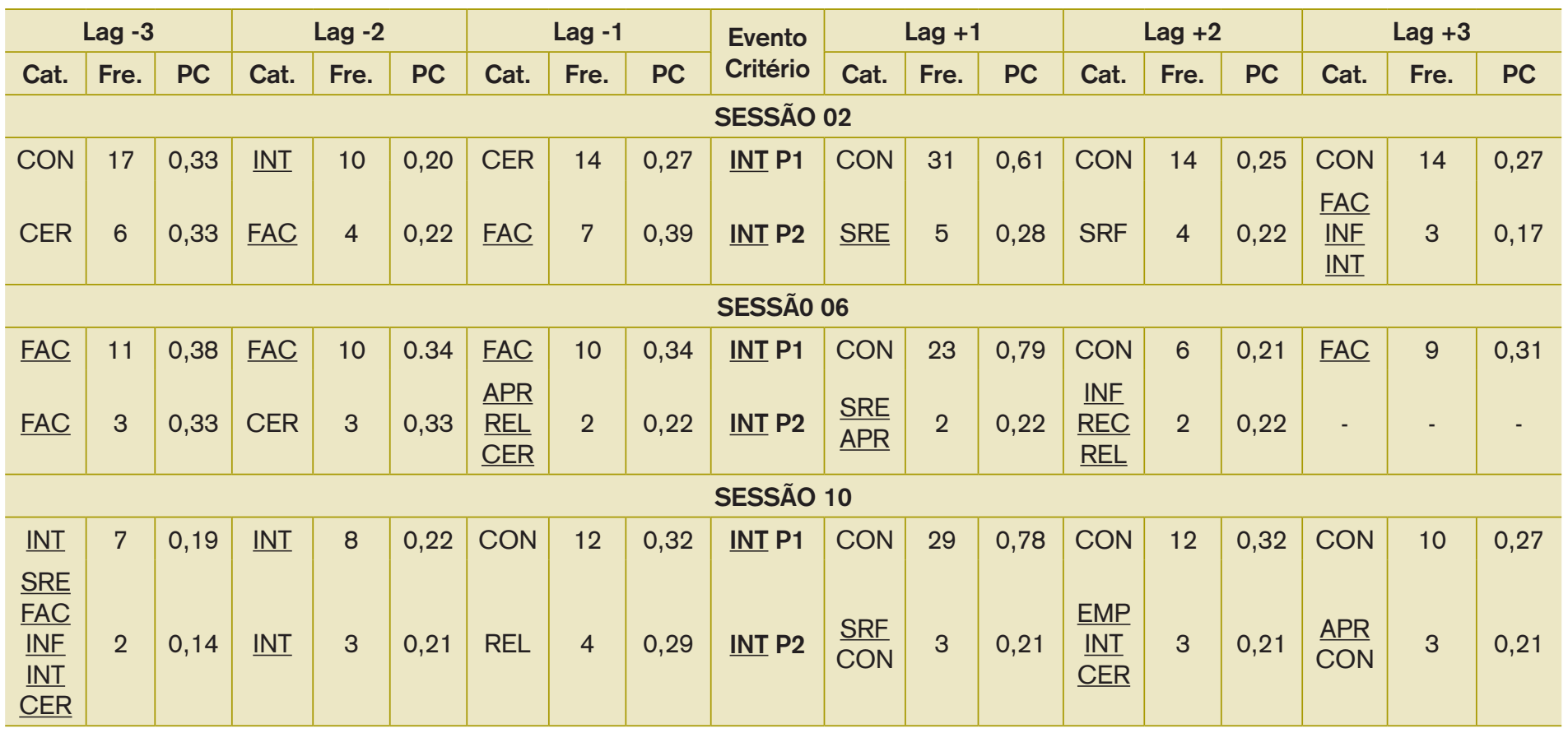

Tabela 3: Categoria (Cat.), Frequência (Fre.), Probabilidade Condicional (PC), Solicita relato (SRE), Facilitação (FAC), Empatia (EMP), Informações (INF), Solicita reflexão (SRF), Recomendação (REC), Interpretação (INT), Aprovação (APR), Relato (REL), Estabelece relações (CER), Concordância (CON). 
Tabela 5. Análises Sequenciais das sessões 2, 6 e 10, para os dois clientes, em seis níveis, Lag -1, Lag - 2, Lag -3, Lag+1, Lag +2 e Lag +3. A categoria de comportamento do terapeuta selecionada como evento critério é Recomendação (REC). As categorias do terapeuta estão grifadas.

\begin{tabular}{|c|c|c|c|c|c|c|c|c|c|c|c|c|c|c|c|c|c|c|}
\hline \multicolumn{3}{|c|}{ Lag -3} & \multicolumn{3}{|c|}{ Lag -2} & \multicolumn{3}{|c|}{ Lag -1} & \multirow{2}{*}{$\begin{array}{l}\text { Evento } \\
\text { Critério }\end{array}$} & \multicolumn{3}{|c|}{ Lag +1 } & \multicolumn{3}{|c|}{ Lag +2} & \multicolumn{3}{|c|}{ Lag +3} \\
\hline Cat. & Fre. & PC & Cat. & Fre. & PC & Cat. & Fre. & PC & & Cat. & Fre. & PC & Cat. & Fre. & PC & Cat. & Fre. & PC \\
\hline \multicolumn{19}{|c|}{ SESSÃO 02} \\
\hline CON & 7 & 0,23 & $\frac{\frac{A P R}{R E C}}{\frac{R O N}{C O N}}$ & 5 & 0,17 & CON & 13 & 0,43 & REC P1 & CON & 13 & 0,43 & CON & 7 & 0,23 & $\underline{\mathrm{INF}}$ & 6 & 0,20 \\
\hline$\frac{\mathrm{INF}}{\mathrm{REL}}$ & 4 & 0,19 & REC & 6 & 0,29 & $\begin{array}{l}\mathrm{SOL} \\
\mathrm{COU}\end{array}$ & 5 & 0,24 & REC P2 & $\mathrm{COU}$ & 6 & 0,29 & $\underline{\mathrm{REC}}$ & 6 & 0,29 & $\mathrm{COU}$ & 8 & 0,38 \\
\hline \multicolumn{19}{|c|}{ SESSÃ0 06} \\
\hline REC & 11 & 0,31 & $\begin{array}{l}\text { CON } \\
\text { SRE }\end{array}$ & 9 & 0,25 & INF & 8 & 0,22 & REC P1 & CON & 16 & 0,44 & CON & 11 & 0,31 & REC & 11 & 0,31 \\
\hline$\underline{I N F}$ & 4 & 0,29 & $\begin{array}{l}\frac{\text { SRF }}{\text { INT }} \\
\underline{\text { APR }} \\
\underline{\text { REC }}\end{array}$ & 2 & 0,14 & INF & 6 & 0,43 & REC P2 & $\frac{\mathrm{INF}}{\mathrm{CER}}$ & 3 & 0,21 & SRF & 3 & 0,21 & REL & 3 & 0,21 \\
\hline \multicolumn{19}{|c|}{ SESSÃO 10} \\
\hline$\frac{\frac{\mathrm{TOU}}{\mathrm{REC}}}{\mathrm{CON}}$ & 2 & 0,13 & REL & 3 & 0,20 & $\frac{\text { APR }}{\mathrm{CON}}$ & 3 & 0,20 & REC P1 & CON & 6 & 0,40 & $\underline{I N F}$ & 3 & 0,20 & CON & 6 & 0,40 \\
\hline REC & 6 & 0,27 & $\underline{\mathrm{REC}}$ & 7 & 0,32 & $\underline{\text { REC }}$ & 6 & 0,27 & REC P2 & $\frac{\mathrm{REC}}{\mathrm{CON}}$ & 6 & 0,27 & $\underline{\text { REC }}$ & 7 & 0,32 & $\underline{\mathrm{REC}}$ & 6 & 0,27 \\
\hline
\end{tabular}

Tabela 4: Categoria (Cat.), Frequência (Fre.), Probabilidade Condicional (PC), Solicita relato (SRE), Informações (INF), Solicita reflexão (SRF), Recomendação (REC), Interpretação (INT), Aprovação (APR), Outras verbalizaçães do terapeuta (TOU), Solicitação (SOL), Relato (REL), Estabelece relações (CER), Concordância (CON), Outras verbalizações do cliente (COU).

Tabela 6. Análises Sequenciais das sessões 2, 6 e 10, para os dois clientes, em seis níveis, Lag -1, Lag - 2, Lag -3, Lag+1, Lag +2 e Lag +3. A categoria de comportamento do terapeuta selecionada como evento critério é Aprovação (APR). As categorias do terapeuta estão grifadas.

\begin{tabular}{|c|c|c|c|c|c|c|c|c|c|c|c|c|c|c|c|c|c|c|}
\hline \multicolumn{3}{|c|}{ Lag -3} & \multicolumn{3}{|c|}{ Lag -2 } & \multicolumn{3}{|c|}{ Lag -1 } & \multirow{2}{*}{$\begin{array}{l}\text { Evento } \\
\text { Critério }\end{array}$} & \multicolumn{3}{|c|}{ Lag +1 } & \multicolumn{3}{|c|}{ Lag +2 } & \multicolumn{3}{|c|}{ Lag +3 } \\
\hline Cat. & Fre. & PC & Cat. & Fre. & PC & Cat. & Fre. & PC & & Cat. & Fre. & PC & Cat. & Fre. & PC & Cat. & Fre. & PC \\
\hline \multicolumn{19}{|c|}{ SESSÃO 02} \\
\hline $\begin{array}{l}\frac{\mathrm{APR}}{\mathrm{REL}} \\
\mathrm{CER}\end{array}$ & 5 & 0,13 & $\frac{F A C}{C O N}$ & 8 & 0,20 & FAC & 12 & 0,30 & $\frac{\text { APR }}{\mathrm{P} 1}$ & CON & 13 & 0,33 & CON & 13 & 0,33 & CON & 8 & 0,20 \\
\hline SRE & 4 & 0,22 & REL & 4 & 0,22 & $\begin{array}{l}\text { REL } \\
\text { CER }\end{array}$ & 4 & 0,22 & $\frac{\text { APR }}{\mathrm{P2}}$ & $\begin{array}{l}\underline{\mathrm{INF}} \\
\underline{\mathrm{INT}} \\
\underline{\mathrm{IOU}}\end{array}$ & 3 & 0,17 & $\begin{array}{l}\text { CER } \\
\text { CON }\end{array}$ & 3 & 0,17 & $\begin{array}{l}\text { FAC } \\
\text { EMP }\end{array}$ & 3 & 0,17 \\
\hline \multicolumn{19}{|c|}{ SESSÃ0 06} \\
\hline$\underline{F A C}$ & 11 & 0,37 & CON & 8 & 0,27 & CON & 20 & 0,67 & $\frac{\text { APR }}{\mathrm{P} 1}$ & CON & 18 & 0,60 & CON & 9 & 0,30 & CON & 7 & 0,23 \\
\hline CER & 5 & 0,24 & SRE & 4 & 0,19 & REL & 7 & 0,33 & $\frac{\text { APR }}{\mathrm{P} 2}$ & $\underline{\mathrm{INF}}$ & 7 & 0,33 & REL & 8 & 0,38 & $\underline{F A C}$ & 5 & 0,24 \\
\hline \multicolumn{19}{|c|}{ SESSÃO 10} \\
\hline $\begin{array}{l}\frac{F A C}{R E L} \\
\text { CON }\end{array}$ & 8 & 0,15 & CON & 9 & 0,17 & REL & 14 & 0,26 & $\frac{\mathrm{APR}}{\mathrm{P} 1}$ & CON & 15 & 0,28 & CON & 11 & 0,21 & CON & 11 & 0,21 \\
\hline$\underline{F A C}$ & 5 & 0,33 & EMP & 4 & 0,27 & $\underline{F A C}$ & 5 & 0,33 & $\frac{\text { APR }}{\text { P2 }}$ & $\underline{\text { SRE }}$ & 4 & 0,27 & $\begin{array}{l}\text { REL } \\
\text { CON }\end{array}$ & 3 & 0,20 & CER & 3 & 0,20 \\
\hline
\end{tabular}

Tabela 5: Categoria (Cat.), Frequência (Fre.), Probabilidade Condicional (PC), Solicita relato (SRE), Facilitação (FAC), Empatia (EMP), Informações (INF), Interpretação (INT), Aprovação (APR), Outras verbalizações do terapeuta (TOU), Relato (REL), Estabelece relações (CER), Concordância (CON). 


\section{Discussão}

Os resultados das análises sequenciais, tendo como comportamento critério as categorias do terapeuta, mostraram alguns padrões de interação, que foram encontrados também em outros estudos. Por exemplo, a categoria Solicitação de Relato-T ser antecedida e sucedida pela categoria Relato-C, corrobora com os dados de correlação de Garcia (2014), dos estudos de análise sequencial de Silveira et al. (2010) e de Sadi (2011).

Outro padrão de comportamento encontrado foi Facilitação-T com Facilitação-T, sendo preciso destacar que de acordo com o protocolo de categorização do SiMCCIT (Zamignani, 2007) a categoria Facilitação-T é caracterizada por verbalizações curtas que ocorrem durante a fala do cliente indicando atenção ao relato e sugerindo a sua continuidade, sendo classificada pelo autor como do tipo evento, isto é, registrada em número de ocorrência, sem intervalo de tempo, diferentemente das demais categorias que são denominadas do tipo estado, possuindo duração (início/fim). Esse aspecto da Facilitação-T permite sua ocorrência repetidas vezes durante uma fala do cliente, pois não necessita a interrupção do tempo para a sua categorização. Assim, o padrão encontrado de Facilitação-T com Facilitação-T demonstra que a terapeuta se utilizou deste recurso seguidas vezes, durante uma mesma fala do cliente, sendo essa análise possível de ser verificada apenas com o recurso da análise sequencial. Também se destacaram com Facilitação-T as categorias Solicitação de Relato-T e Relato-C, o que mostra o cuidado da terapeuta em construir um ambiente acolhedor que favorecesse o relato do cliente.

Tais padrões de interação em relação à categoria Facilitação-T evidenciam o tipo de intervenção aplicada, pois diante de universitários com TAS que apresentam dificuldade em se expressar pela timidez excessiva, demandam uma intervenção da terapeuta para esse déficit comportamental. Assim, a terapeuta contribui para a melhora dos clientes estimulando o relato, uma vez que a conversação é de suma importância para as interações sociais (Del Prette \& Del Prette, 2010; Beidel, 2014).

O padrão de comportamento do terapeuta em estimular verbalizações dos clientes no processo terapêutico individual, é avaliado como vantajoso por
Rocha et al (2012), uma vez que possibilitou ensaios extensos e com repetição. Dessa maneira, a terapeuta teve melhor aproveitamento para modelar habilidades específicas relacionadas aos objetivos terapêuticos, diferentemente do tratamento em grupo, que foi rejeitado pelos clientes. $\mathrm{O}$ atendimento individual tornou o ambiente terapêutico seguro para a emissão de novas respostas, concordando com o estudo de Garcia-Lopez et al (2015) que ressaltou a importância do ambiente acolhedor para que o fóbico social consiga se expressar na terapia.

A categoria Facilitação-T ocorreu antes e após a categoria Relato- $\mathrm{C}$ em maior frequência para $\mathrm{P} 1$ do que para P2. Essa diferença entre os clientes pode ser entendida considerando as características de cada um dos casos, descritos em Rocha (2012). Nos estudos de caso de cada participante o P1 apresentava maior dificuldade em relatar, demonstrando um repertório de entrada restrito. Esse dado, analisado em conjunto com as análises sequenciais encontradas, atesta a flexibilidade do procedimento utilizado (Bolsoni-Silva, 2009), que recomenda sua adaptação de acordo com cada cliente, tendo por base, seus déficits/excessos/reservas de repertórios. Assim, $\mathrm{P} 1$ precisava de mais Facilitação-T, para o aumento/instalação do repertório de Relato-C, diferentemente de P2, que já apresentava maior frequência de ocorrência, de acordo com sua descrição no estudo de caso (Rocha, 2012). Essa análise possibilita a inferência de que os estudos de Lags também podem avaliar a coerência do procedimento teórico utilizado com sua execução de fato e, consequentemente, comprova que a intervenção adotada é coerente com os princípios da PBE (APA, 2006), pois o procedimento é flexível e ajustado às queixas, demandas e dificuldades dos clientes.

Esse padrão Facilitação-T - Relato-C também foi encontrado no estudo realizado por Sadi (2011), onde a autora colocou como hipótese de que essa sequência poderia estar envolvida em uma sequência maior, a de Relato-FacilitaçãoRelato. Na presente pesquisa, com análise de três níveis antecedentes e três consequentes, a hipótese pôde ser demonstrada na análise de uma sessão para o cliente P2. Diferentemente em Xavier et al (2012) que realizou análise de probabilidade em uma intervenção com crianças, os dados mostraram a categoria Empatia-T após o Relato-C de 
comportamentos problema, mas da mesma forma, relacionando o manejo do terapeuta ao ambiente acolhedor ao cliente.

No estudo de Raustan (2013) um padrão parecido pôde ser identificado, em que o terapeuta utilizou o silêncio diante da fala dos integrantes do grupo, para não interromper os relatos e, dessa maneira demonstrou atenção. O terapeuta também tinha comportamentos empáticos diante de relatos de comportamentos de apoio emocional. Portanto, o manejo do terapeuta em facilitar o relato do cliente, foi encontrado em outros estudos, e foi interpretado como uma forma do terapeuta estimular o relato do cliente, demonstrando atenção. Assim, na perspectiva da clínica analítico comportamental, um ambiente acolhedor favorece o processo terapêutico (Silvares \& Gongora, 1998) e o terapeuta deve-se constituir como uma audiência não punitiva (Skinner, 1953), sendo que ao se utilizar da categoria de Facilitação-T, o terapeuta buscou a construção deste ambiente acolhedor como evidenciado nas Lags tendo Facilitação-T como critério.

Para a sequência em relação à categoria critério Informação- $\mathrm{T}$, a categoria Concordância-C ocorreu como antecedente e subsequente reiteradamente nas sessões 2 e 6 para um dos clientes (P1). No estudo de Zamignani (2007) foi possível notar essa sequência Informação-Concordância. No estudo de Garcia (2014) a categoria Concordância-C foi uma das categorias em que para o mesmo cliente P1 teve uma média significativamente maior do que do outro cliente (P2), o que corrobora com a diferença também encontrada na análise sequencial do presente estudo.

Sobre as características dos clientes, ambos apresentavam dificuldades de expressão, porém existiram diferenças entre eles, $\mathrm{P} 2$ diferentemente de P1, evitava contato visual e respondia às questões do terapeuta com respostas furtivas, o que mudou com a intervenção, P2 passou a manter o contato visual e a relatar as situações com mais detalhes, como também passou a se envolver nas atividades de ensaio comportamental (Rocha, 2012). Esse padrão de comportamento mais inibido de P2 pode explicar a diferença nas sequências de comportamento, em que P1 apresentou a categoria Concordância após um comportamento do terapeuta, demonstrando entendimento e aten- ção ao terapeuta, tanto como frequência (Garcia, 2014) como na análise sequencial. Outra hipótese é de que as dificuldades de $\mathrm{P} 2$ demandaram do terapeuta maior utilização de outros recursos/técnicas, uma vez que há nas Lags encontradas, uma variedade de procedimentos do terapeuta (antecedentes e subsequentes), conforme descrito na Tabela 3. Assim, o terapeuta reagia de forma diferente ao prestar informações diante de P2. Esses resultados diversos para os clientes, mesmo ambos realizando o mesmo procedimento de intervenção semiestruturada, mais uma vez evidencia que está de acordo com os princípios da Psicologia Baseada em Evidência (APA, 2006).

Da mesma forma, a categoria Concordância-C também ocorreu após a categoria critério Interpretação- $\mathrm{T}$ para o cliente $\mathrm{P} 1$. Tanto Interpretação- $\mathrm{T}$ quanto Concordância-C, são categorias que apresentaram diferenças significativas para os clientes nas análises de Garcia (2014), pois para P2 a frequência da categoria Interpretação-T foi menor, o que pode explicar a diferença apresentada nas análises sequenciais.

No estudo de correlação de Silveira et al. (2010) ocorreu a correlação positiva entre Interpretação- $\mathrm{T}$ e Concordância-C. Em Garcia (2014) para P1 a categoria Interpretação-T apresentou correlação com a categoria Gestos de Concordância-C, apesar de ser uma categoria não vocal, essa categoria apresenta como função a concordância e compreensão com a fala do interlocutor, assim como na categoria vocal (Zamignani, 2007). Ainda tendo Interpretação- $\mathrm{T}$ como categoria critério, no presente estudo, em P2 a categoria Facilitação-T ocorreu como antecedente, diferentemente de para P1, o que pode ser relacionado novamente pela característica do cliente P2, de ter mais dificuldade na interação com a terapeuta, dessa forma, ao emitir Facilitação-T antes de Interpretação-T, a terapeuta promoveu um ambiente acolhedor e ainda estimulou o cliente na sua interação com ela.

Apesar do uso do mesmo banco de dados de Garcia (2014) as análises de correlação ora concordam e ora destoam das encontradas na presente pesquisa. Tais achados destacam a complexidade do estudo da interação terapêutica e indicam que métodos diferentes podem ser complementares na obtenção de resultados e, em conjunto, facilita- 
rem a compreensão do processo terapêutico, que é muito complexo. A literatura carece de estudos que avaliam a interação terapeuta/cliente comparando métodos de análise, que possibilitaria o desenvolvimento do campo e uma discussão da forma mais adequada de estudar o referido fenômeno.

A partir da categoria critério Recomendação-T pode-se destacar a categoria Concordância-C para o P1, que ocorreu como Lag +1 para todas as três sessões, o que sugere a compreensão/concordância com a recomendação do terapeuta. Tal fato também apareceu expresso nas demais Lags de comportamentos subsequentes, com a presença da mesma categoria Concordância- $\mathrm{C}$, além de outras categorias do terapeuta, como informar e recomendar. Sendo que recomendar, também foi significativo para $\mathrm{P} 2$.

Como evento antecedente da categoria critério Recomendação- $\mathrm{T}$, destaca-se o comportamento de Informação-T. Em Silveira et al. (2010) também ocorreu a categoria Informação- $\mathrm{T}$ como precedente de Recomendação-T em análises sequênciais. Diferentemente de Silveira et al. (2010) a categoria Aprovação-T não apareceu como subsequente. Já no estudo de Sadi (2011) Concordância-C ocorreu após Recomendação- $\mathrm{T}$, apesar de essa sequência ocorrer com baixa frequência corrobora com os resultados da presente pesquisa.

O terapeuta informar antes de fazer uma recomendação mostra-se coerente na perspectiva analítica comportamental, pois a categoria Informação-T é definida pelo terapeuta informar ou relatar eventos ao cliente com intenção explicativa. Uma subcategoria de Informação-T é a Justificativa de Intervenções, em que o terapeuta explica ou justifica suas intervenções (Zamignani, 2007), dessa maneira, os resultados evidenciaram que a terapeuta explicava e justificava antes de fazer uma Recomendação-T ao cliente, aumentando assim a probabilidade maior engajamento do mesmo na atividade recomendada.

Para a categoria Aprovação-T como critério, as categorias Relato-C, Estabelece Relações-C e Facilitação-T ocorreram como antecedente à Aprovação-T, e a categoria Concordância-C como consequente. Em relação as categorias que antecedem Aprovação-T, está de acordo com o estudo de Silveira et al. (2010) onde essas categorias também apareceram como antecedentes, com exceção da categoria Facilitação-T que não teve ocorrência. A categoria Facilitação-T está relacionada à categoria Relato-C, dessa forma, pode ser explicada a ocorrência como antecedente de Aprovação- $\mathrm{T}$.

A categoria Aprovação-T após Relato-C também pode ser encontrada em Ruiz-Sancho et al (2015), os achados desta pesquisa mostraram que o terapeuta buscou informações do cliente e, após o relato do cliente, aprovava para posteriormente fazer novamente outra pergunta, o que mostra manejo de reforçar comportamentos de acordo com o relato do cliente. Outro aspecto relacionado à Aprovação- $T$ após o Relato- $C$, sugere que o terapeuta é capaz de selecionar aspectos relevantes do comportamento do cliente, promovendo autoconhecimento, que será útil na elaboração de novo repertório, estando de acordo com a atuação do terapeuta comportamental (Villani, 2002).

Dados da interação terapêutica tanto em medidas de frequência e correlação em Garcia (2014), como na análise sequencial no presente estudo, apresentaram fatores em comum, como também diferenças entre os padrões de comportamento do terapeuta em relação aos clientes. As diferenças, mostraram que a terapeuta se comportava de acordo com cada cliente, considerando suas dificuldades e suas reservas comportamentais particulares, dessa forma, demonstra que a flexibilização do terapeuta na forma com que se relaciona com cada cliente foi essencial, uma vez que a avaliação da psicoterapia demonstrou êxito para ambos os clientes.

\section{Considerações Finais}

Com a presente pesquisa pretendeu-se estudar a interação terapêutica em uma terapia analítico comportamental para universitários com transtorno de ansiedade social. A partir de análises sequenciais foi possível inferir alguns padrões de comportamentos do terapeuta e do cliente e relações de dependência entre eles.

Foi possível destacar algumas sequências de comportamentos relevantes para a prática do terapeuta que corroboraram com a literatura, entre elas destaca-se a sequência Solicitação de Relato-T - Relato-C, Facilitação-T - Relato-C, Concordância-C - Informação-T - 
Concordância-C, Solicita Reflexão-T - Estabelece Relações-C - Facilitação-T. Tais relações destacam aspectos do processo terapêutico que demonstraram como o terapeuta pode se comportar na interação terapêutica, bem como de que forma o cliente responde e, assim, compreender a ocorrência de classes de respostas e de estímulos, relacionando com a queixa dos casos atendidos.

Esta pesquisa pode contribuir para a formação de terapeutas comportamentais, na medida em que demonstrou a forma com que uma terapeuta experiente aplicou um procedimento análise comportamental associado ao treino de habilidades sociais para casos de fobia social, mapeando todo o processo via análise sequencial. Assim, hipotetiza-se que diante de um diagnóstico de TAS, o terapeuta deve ser acolhedor, facilitar o relato do cliente e ao mesmo tempo aprovar aspectos desse relato que são relevantes, garantindo flexibilidade conforme demandas individuais.

Algumas limitações neste estudo devem ser destacadas. Uma delas é de que não foi aplicada a análise sequencial em todas as categorias previstas no SiMCCIT como evento critério. Outra limitação foi a utilização de três sessões para a análise ao invés de todas as sessões do atendimento. Dessa forma, novas pesquisas poderão abranger outras categorias critérios de comportamentos da interação terapêutica, como também aplicar a análise sequencial em um número maior de sessões, e ainda realizar a análise para outros transtornos psiquiátricos.

\section{Referências}

American Psychiatric Association (2002). DSMIV-TR Manual de Diagnóstico e Estatística das Perturbações Mentais, 4. ed. Consultoria e coordenação de Miguel R. Jorge. Tradução: Cláudia Dornelles. Porto Alegre: Artmed.

American Psychiatric Association (2013). Diagnostic and Statistical Manual of Mental Disorders, Fifth Edition. Washington, DC: American Psychiatric Association.

American Psychiatric Association (2006). Evidence-Based Practice In: Psychology. American Psychologist. 271-285.

Araújo, A. C. \& Neto, F. L. (2014). A Nova Classificação Americana Para os Transtornos Mentais - o DSM-5. Rev. bras. ter. comport. cogn., São Paulo, v. 16, n. 1.

Bakeman, R. \& Gottman, J. M. (1997) Observing Interaction: An Introduction to Sequential Analysis. New York: Combridge university Press.

Barkowski, S., Schwartze, D., Strauss, B., Burlingame, G. M., Barth, J., Rosendahl, J. (2016). Efficacy of group psychotherapy for social anxiety disorder: A meta-analysis of randomized-controlled trials. Journal of Anxiety Disorders, 39, 44-64.

Batista, et. al. (2012) social phobia in Brazilian university students: Prevalence, under-recognition and academic impairment in women. Journal of Affective Disorders, 136, 857-861.

Beidel, D. C., Alfano, C. A.; Kofler, M. J.Rao, P. A. Scharfstein, L. \& Wong Sarver, N. (2014). The impact of social skills training for social anxiety disorder: A randomized controlled trial. Journal of Anxiety Disorders, 28, 908-918.

Del Prette, A. \& Del Prette, Z. A. P. (2010). Habilidades sociais e análise do comportamento: Proximidade histórica e atualidades. Perspectivas em Análise do Comportamento, 1, 104-115.

Garcia, V. A. (2014). Análise da interação terapêutica em intervenções com universitários com transtorno de ansiedade social. 123f. Dissertação (Mestre em Psicologia do Desenvolvimento e Aprendizagem) - Unesp, Faculdade de Ciências, Bauru. 
Bolsoni-Silva, A. T. (2009). Habilidades sociais de universitários: procedimentos de intervenção na perspectiva da Análise do Comportamento. In: Wielenska, R. C. (Org.). Sobre Comportamento e Cognição: Desafios, soluções e questionamentos. Santo André: ESETec Editores Associados, p. 21-52.

Garcia-Lopez, L-J. Salvador, M do C. \& Reyes, A. de Los (2015) Assessment of Social Anxiety in Adolescents. In: Ranta, K. et al. (eds.) Social Anxiety and Phobia in Adolescents: Development, Manifestation and Intervention Strategies, 121-122.

Meyer, S. B. (2009). Análise de 'solicitação de informação' e 'recomendação' em banco de dados de terapias comportamentais. $328 \mathrm{f}$. Tese (LivreDocência em Psicologia clínica) - Instituto de Psicologia, Universidade de São Paulo. São Paulo.

Rocha, J. F. (2012). Efeitos de uma intervenção comportamental com treino de habilidades sociais para universitários com fobia social. 156f. Dissertação (Mestrado em Psicologia do Desenvolvimento e Aprendizagem) Faculdade de Ciências, Universidade Estadual Paulista "Júlio de Mesquita Filho", Bauru.

Rocha, J. F.; Bolsoni-Silva, A. T. \& Verdu, A. C. M. A. (2012) O uso do treino de habilidades sociais em pessoas com fobia social na terapia comportamental. Perspectiva em análise do comportamento, 3(1), 38-56.

Roustan, M., Rodríguez, C. I., \& Argilaga, T. A. (2013) Sequential Analysis of an interactive peer support group. Psicothema, 25(3), 396-401.

Sadi, H. M.(2011) Análise dos comportamentos de terapeuta e cliente em um caso de Transtorno de Personalidade Boderline. 120 f. Tese (Doutorado em Psicologia Clinica) Instituto de Psicologia, Universidade de São Paulo. São Paulo.

Silvares, E. F. M., \& Gongora, M. A. N. (1998). Psicologia clínica comportamental: a inserção da entrevista com adultos e crianças. São Paulo: EDICON.

Silveira, F. F., Bolsoni-Silva, A. T. \& Meyer, S. B. (2010) Therapist's directive and nondirective behavior: analysis of their effects in a parent training group. International Journal of Behavioral Consultation and Therapy 6(2), 124-133.
Skinner, B. F. (1957). Verbal behavior. NewYork, NY: Appleton-Century-Crofts.

Skinner, B. F. (2007). Ciência e Comportamento Humano (J. C. Todorov, \& R. Azzi, Trads.,11 $1^{\mathrm{a}}$ ed.). São Paulo: Martins fontes (Obra original publicada em 1953).

Villani, M. C. S. (2002). Considerações sobre o desempenho do terapeuta comportamental. In: Teixeira, A. M. S. (org.) et al. Ciência do Comportamento: conhecer e avançar. São Paulo: ESETEC, 27-33.

Xavier, R. N., Kanter, J. W., \& Meyer, S. B. (2012). Transitional Probability Analysis of Two-Child Behavior Analytic Therapy Cases. International Journal of Behavioral Consultation Therapy, $7(2-3), 182-188$.

Zamignani, D. R. (2007) O desenvolvimento de um sistema multidimensional para a categorização de comportamentos na interação terapêutica. 289 f. Tese (Doutorado em Psicologia Clínica) - Instituto de Psicologia, Universidade de São Paulo. São Paulo. 2007.

Zamignani, D. R. \& Meyer, S. B. (2011) Comportamentos verbais do terapeuta no sistema multidimensional para a categorização de comportamentos na interação terapêutica. Revista Perspectivas, 2(1), 25-45.

\section{Informações do Artigo}

\section{Histórico do artigo:}

Submetido em: 25/07/2016

Primeira decisão editorial: 16/09/2016

Aceito em 05/11/2016

Editor associado: Nicodemos Batista Borges 